\title{
Analysis of Security in Wireless Network: A Survey
}

\author{
Mr. Abhimanyu. D. Sangale ${ }^{a, 1}$, Dr. Sanjeev Kumar Sharma ${ }^{a}$ \\ ${ }^{a}$ Dept of CSE, Oriental University, Indore, India.
}

\begin{abstract}
Information security maintenances with the truthfulness, accessibility, and secrecy of knowledge irrespective of the arrangement of the information might take. Data security is frequently branded in different ways like in connected and non-connected security. Non-connected grid security varies with execution prerequisites. The proposed study of worries and safety mechanism stages accessible in non-connected networks. The anticipated representations for nonconnected networks are grounded on diverse significances for sanctuary and effortlessness.
\end{abstract}

Keywords. Security maintenance, non-connected network, data security.

\section{Introduction}

Sanctuary in non-connected devices means to protect the information or the data from the unauthorized access, discloser, usage, alteration, disturbance, scrutiny, inspection, recording or obliteration. The difference of opinion like computer security, knowledge assurance or guarantee and information security are constantly used in a mixed way or interchangeably. These turfs are interconnected often and stake the mutual goal for shielding the secrecy, truthfulness and accessibility of the info packets, nevertheless there is certain difference between them. [1]

There seems to be a great deal of proprietary knowledge accessible to military, federal departments, administrative offices, hospitals, corporate offices as well as several large enterprises about their staff, clients, goods, analysis and financial status. All of this evidence is collected in a digital format and sent to other organizations throughout the network. So, these days wireless communication is used to transmit this sensitive data, which makes it easy target to other organizations to hack it or modify the data. This paper provides an introduction to assaults as well as the vulnerability of access point in the non-connected network.

\footnotetext{
${ }^{1}$ Mr. Abhimanyu. D. Sangale, Dept of CSE, Oriental University, Indore, India

Email: abhimanyu.sangale@gmail.com.
} 


\section{Security Policy in Wirelessnetwork}

Non-linked networks aid as equivalent as wired network. In wireless network, as the data is mid-air data transmission, its more susceptible for attacks and illegal uses. So, to protect this data, higher security system needs to be implemented, which is come with lofty price tags. To keep the benchmark, companies have to pay against their operational needs and use the non-connected connectivity. Several kinds of safe guard strategies are also used to secure the wireless network. In sight of each wireless network backdoor, setting up a series of protection plans would essentially prevent security problems such as illegal terminal entry, bogus access points, midway data interception, etc. [2]

\subsection{Distinct types of wireless communication attacks:}

Often, wireless attacks are categorized as aggressive, passive, near, insider intrusion and service provider assault. Personal data systems and networks provide tempting opportunities and should be prone to diseases from the wide spectrum of malicious hackers, from ransomware to nation-state attackers. There should be a framework that reduces harm and gets better soon when incidents happen. [3]

\subsection{Five varieties of attacks:}

Aggressive attack: A aggressive attack searches unencrypted traffic and finds confidential data and clear-text codes that can be used in other types of attacks. Traffic collection, insecure communications recording, weakly encrypted traffic decryption, and information capture of password-like authentication are passive risks. Passive network process interception helps adversaries to see future actions. Without the permission or knowledge of the user, passive attacks end in the transmission of information or data files to an attacker.

Active Attack: The intruder seeks to circumvent or hack through secured networks in an active assault. With stealth, malware, worms, or Trojan horses, this can be achieved. Active threats affect attempts to disable or crack security features, implement malicious code, and steal or change data. These attacks are installed against a network backbone, exploit data in transit, electronically break an enclave, or threaten an authenticated remote user during an attempt to connect to an enclave. Discovery or distribution of data files, DoS or alteration of active attack material.

Disseminated Attack: A disseminated intrusion allows the competitor to add malware to a "trusted" component or software, a Trojan horse or back-door program, for example, which will later be spread to several other organizations and clients. Distribution attacks in the warehouse or during distribution depend on the malicious exploitation of hardware or software. These threats cause security vulnerabilities in order to gain unauthorized access at a later date to a sensitive or device feature, such as a back door to a product [4].

Internal Attack: An internal attack includes somebody from the within, including a disgruntled former employee. It may be harmful or not harmful to attack the system malicious activities. Intentionally, dishonest insiders eavesdrop, intercept, or destroy information; use information in a dishonest manner; or deprive all authorized user's 
entry. Usually, no malicious attacks arise from carelessness, lack of information, such purposes as executing a mission, deliberate circumvention of privacy.

Close-in Attack: A near-in attack requires someone trying to get physically close to network elements, data, and systems in order to learn more about a network. In order to change, collect, or deny access to information, close-in attacks consist of ordinary individuals who achieve close physical proximity to networks, devices, or services. Unrestricted access or both through surreptitious intrusion into the network, or both, close physical proximity is accomplished. [5]

\section{Security Goles for Wirelss Network}

The From this list, we can use different categories of stages that are compatible with our need for protection and utility.

Stage 1 Security: Security at the primary stage is necessary and is translated into every wireless system that may be bought today. It is backed by a 'Wired Equal Privacy' algorithm that is intended to beat most security threats. Details can only be decrypted by the receiver with the right WEP key. Furthermore, WEP is not used to block unintended wireless network connectivity. WEP (wired equivalent privacy): It gives two main elements of defines (authentication and confidentiality).

WEP used a joint key feature in addition encryption of the RC4 algorithm and used CRC-32's consistency confirmation. Initially, WEP tried to use four data encryption operations, initially 24 - bit vector combination behaves as encoding or decoding just like the key used in 40 - bit WEP algorithm.

The initiation of a virtual-random number originator serves as resulting key (PRNG). Second, the honesty of the algorithm is shown by the plaintext and again concatenates with the plaintext. The main sequence and ICV results will be handled by the RC4 algorithm. By adding the IV ahead for the encoded text, the final encryption message is generated. WEP tries to decrypt five operations by using them.

The pre shared key (PSK) and the iteration vector (IV) were initially merged to form a hidden key. Then, the encoded text and concealed key are present in the CR4 algorithm, resulting in a plaintext. Thirdly, it can distinguish the ICV and plaintext. Lastly, Integrity Algorithm gets plaintext to form a replacement ICV and eventually the latest ICV is matched with old ICV.

WEP feebleness: Imitation of packets can't be vetoed by using WEP. Repetitional attacks can't be vetoed. Attackers can simply record and replay packets as desired and that they are going to be acknowledged as authentic. RC4 used by WEP is inappropriate. Very feeble key is used, in hours to minutes, using readily available tools such as 'air cracking' might be over forced on regular systems. WEP reuses vectors for initialization. Without understanding the encryption key, a dispersion of available cryptanalytic methods will decrypt data. WEP allows an attacker without understanding the encryption key to undetectably change a message.

However, even with stage 1 protections, there are still many unresolved security risks remaining within a wireless network system.

Stage 2 Security: In stage 1 security, there are some treats or its not completely secure. Its easily compromised. 
3.1. Easy Access: If adequate security procedures are not enforced on the network, non-connected LANs are incredibly informal to find and join. Attackers will invade the network without having to enter the facility physically. If the SSIDs are transmitted completely through the system, they could be stopped and enable unsanctioned access (Secure Device Identifiers are allocated to a wireless network).

3.2. Rough access points: In an organization if an access point is installed without the permission of administrator are called rough access point. Access points are most conveniently bought anywhere and mounted. However, appropriated security measured would not be enforced on the system, depending on the person upgrading the access points, fixing an entrance point for attackers and hackers. [7]

3.3. Eavesdropping: It says that, it's an intervention over the non-connected networks of the evidence being communicated. Eavesdropping is also achieved by wireless sniffers, such as software for air dumping.

3.4. Traffic analysis: Facilitates the collection of data transfer and network operation information through monitoring/intercepting wireless communication patterns.

3.5. Data tampering: This explains the possibility that wireless data is frequently collected and discarded during transmission.

3.6. Denial of service (DoS): The communication channel will be chunked by the intruders by using a powerful frequency generator, thus disturbing access to the network.

Stage 3 Security: In Stage 2 security, there are still several risks. Using WPA (Wi-Fi Safe Access), WPA facilitates increased regulation of network access, enhances encryption technologies, and enforces data integrity. Without the users needing to modify the hardware, the WPA arrived with the intention of solving the problems inside the WEP cryptography process. WPA boosts software/firmware over WEP (no new hardware required).

3.1 Personal WPA or WPA-PSK: It is used for domestic usage authentication in SOHO (small office/home office). Personal WPA does not use a server for authentication, so the data encryption key can be up to 256 bits. Keys are often any alphanumeric string and are only used to barter the initial access point session (APs). Both the app and the AP also have this key here. WPA offers reciprocal authentication, meaning the keys are never sent over the air.

3.2 Enterprise WPA: An 802.1x authentication server is formed during this method of authentication mode, generating superb access and protection within the users' wireless network traffic. For authentication, this WPA uses 802.1X+EAP (Extensible Authentication Protocol) but replaces WEP with the more sophisticated TKIP (Temporary Key Incorporation Protocol) encryption again. There is no Pre-Shared (PSK) key used here. TKIP uses the RC4 strategy in an analogous WEP but produces a hash before the RC4 algorithm improves. A duplication of the initialization vector is generated. [8]

One copy is shipped to subsequent step, and therefore the other is hashed (mixed) with the bottom key. After performing the hashing, the result generates the key to the 
package that's getting to join the primary copy of the initialization vector, occurring the increment of the algorithm RC4. Then, from the text you just want to cryptography, there is the generation of a sequential key with an XOR, then generating the cryptographic text. Lastly, the message is ready to be received. By inverting the operation, the encryption and decryption will be carried out.

Stage 4 Security: Some more threats and weakness in stage 2 security.

3.7. Encryption vulnerabilities: WPA has certain encryption vulnerabilities; code tampering and masquerading are still not entirely fixed by protection stage 2 .

\subsection{Compromising performance: device performance}

degrades and data transfers and communication speeds are dropped due to intense authentication and encryption protocol computations.

\section{Recently Discovered Insecurities in Wireless Network}

The 'hole 196' flaw recently found in the WPA2 authentication protocol opened WPA2secured Wi-Fi networks to insider attacks. This helps insiders to send secure group addressed data traffic using the Group Temporary Key. This data traffic was only meant to be conveyed by Access Point, not by client nodes. But if this GTK traffic is submitted by a malicious insider, he/she can be prepared to upgrade other ARP cache nodes with the aid of ARP request broadcast with GTK. This allows the insider to scent all the user's private information. [9]

\section{Variables Addressed During Wireless Network Architecture}

We would like to learn about the considerations below when erecting a wireless network in order to design a secure wireless network.

Concentrate on the physical position of the connection point: within the breeze, the wireless signal is spreading; its border definitely does not seem obvious because of the wired network; at any time, the signal will appear beyond the exact coverage. With respect to physical secure, the location of the AP must be measured by means of a special purpose method. We ought to reduce the probability of disclosure of cellular signals beyond the coverage of the network as much as possible.

Access Point control and Management: During a wireless network, where there are multiple APs, the way to control the maintenance and hence the tracking of such APs seems to be highly significant. If there is no cost-effective AP control scheme, the network protection void will be triggered and the attacker will be able to interrupt.

The verification and user identification: While constructing a wireless network, the authentication of wireless network connections to wired network services must be taken into account. To ease user management, enterprise IT network administrators can incorporate the wireless local area network into the existing RADIUS building. It also helps to classify remote consumers in large- scale enterprises. [10] 


\section{Conclusion}

The paper presents numerous security policies in various wireless networks, different from their wireless network and their security concern. Each network has to deliver different protection problems thanks to different features of different networks. Thanks to general security priorities, though, there are several common concerns. This is why a common algorithm is always generated to defend these numerous wireless networks.

\section{References}

[1]. Bodhe, Abhijit, Mayur Masut and A. S. Umesh." Wireless LAN security at tacks and CCM protocol with some best practices in deployment of services." International Research Journal of Engineering and Technology (IRJET) 3.1 (2016): 429-436.

[2]. Umesh, Abhijit S. Bodhe1 Dr AS." Rouge Access point:A Threat to Wireless Society."

[3]. Bodhe, Abhijit S., and N. Jagdisha." Security Enhancement Scheme in Mobile Wireless Sensor Networks Using RAPD Approach." 2019 Third International conference on I-SMAC (IoT in Social, Mobile, Analytics and Cloud) (I-SMAC). IEEE, 2019.

[4]. B.Daya, (2013) "Network Security History, Importance, and future" International Journal of Advance Foundation and Research in Science \& Engineering, Volume 1, Issue 3.

[5]. Bodhe and A. Sangale," Network Parameter Analysis; ad hoc WSN for Security Protocol with Fuzzy Logic," 2020 Second International Conference on Inventive Research in Computing Applications (ICIRCA), Coimbatore, India, 2020, pp. 960-963, doi: 10.1109/ ICIRCA48905.2020.9183311.

[6]. http://interscience.in/IJSSAN Vol1Iss1 Zpaper25.pdf

[7]. Potharaju, S. P., \& Sreedevi, M. (2018). A novel cluster of quarter feature selection based on symmetrical uncertainty. Gazi University Journal of Science, 31(2), 456-470.

[8]. http://www.airtightnetworks.com/WPA2-Hole196

[9]. http://www.wifi.org/knowledge_center/ wpa2

[10]. Potharaju, S. P., Sreedevi, M., \& Amiripalli, S. S. (2019). An Ensemble Feature Selection Framework of Sonar Targets Using Symmetrical Uncertainty and Multi-Layer Perceptron (SUMLP). In Cognitive Informatics and Soft Computing (pp. 247-256). Springer, Singapore.

[11]. S. Alabady, (2009) "Design and Implementation of a Network Security," Technology, Vol. 1,p. 11.

[12]. Jain, M.K., 2011. Wireless sensor networks: 10. Ning, P., A. Liu and W. Du, 2008. Mitigating DoS Security issues and challenges. International Journal attacks against broadcast authentication in wireless of Computer and Information Technology, sensor networks. ACM Transactions on Sensor 2(1): 62-67

[13]. Dr. Gurjeet Singh (April 2012) "Security Issues in Wireless Broadband Networks" Volume 12 Issue 5 Version 1.0, Double Blind Peer Reviewed International Research Journal.

[14]. Sikkens B. (2008). Security Issues and proposed solutions concerning authentication and authorization for WiMax. 8th twenty student Conference on IT. 